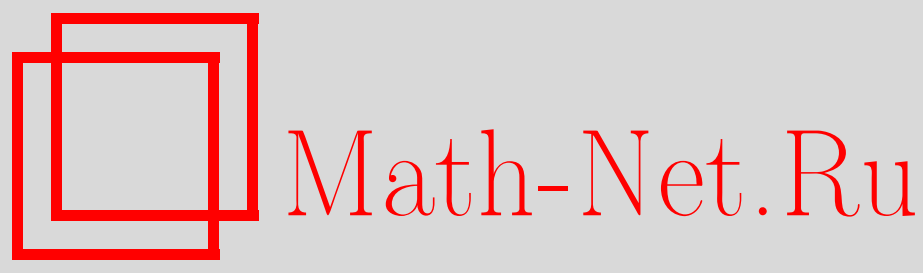

И. В. Фомин, Модели космологической инфляции в кинетическом приближении, ТМФ, 2017, том 191, номер 2, 354-365

DOI: https://doi.org/10.4213/tmf9177

Использование Общероссийского математического портала Math-Net.Ru подразумевает, что вы прочитали и согласны с пользовательским соглашением http://www.mathnet.ru/rus/agreement

Параметры загрузки:

IP : 3.89 .197 .203

26 апреля 2023 г., 11:58:48

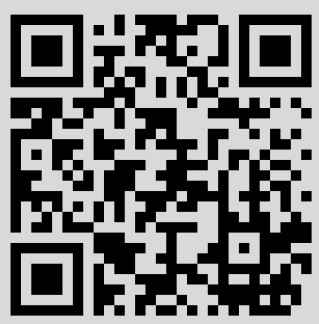


Том 191, № 2

май, 2017

(C) 2017 г.

\author{
И. В. Фомин*
}

\title{
МОДЕЛИ КОСМОЛОГИЧЕСКОЙ ИНФЛЯЦИИ В КИНЕТИЧЕСКОМ ПРИБЛИЖЕНИИ
}

\begin{abstract}
Рассматривается построение моделей космологической инфляции с приближенной линейной зависимостью кинетической энергии скалярного поля от параметра состояния. Проводится сопоставление полученных решений с известными космологическими моделями и рассчитываются основные параметры космологических возмущений.
\end{abstract}

Ключевые слова: инфляция, скалярное поле, космологические возмущения.

DOI: https://doi.org/10.4213/tmf9177

\section{1. ВВЕДЕНИЕ}

Теория космологической инфляции успешно описывает состояние ускоренного расширения Вселенной на ранних стадиях эволюции [1], [2]. Также инфляционная космология объясняет происхождение первичных неоднородностей и предсказывает их спектр. Механизм начального инфляционного сценария и повторного ускоренного расширения можно описать в предположении существовании скалярного поля $\phi-$ инфлатона, определяющего стадию инфляции и поле квинтэссенции, которое является источником наблюдаемого ускоренного расширения Вселенной [3]-[5]. Различные инфляционные сценарии отличаются выбором эффективного потенциала $V(\phi)$.

Скалярные поля играют фундаментальную роль в космологии, задавая уравнение состояния $p=w \varepsilon$, где $p$ - давление, $\varepsilon$ - плотность энергии и $w$ - параметр состояния, в виде $p \approx-\varepsilon$, что приводит к ускоренному расширению Вселенной на ранней стадии эволюции [6]. Хотя первоначально инфляционные модели были предложены, чтобы обеспечить решение проблем сингулярности, плоского пространства, горизонта, проблемы однородности и отсутствие магнитных монополей, на сегодняшний день наиболее полезным свойством инфляции является то, что она порождает возмущения плотности, определяющие распределение материи во Вселенной, что можно проверить различными способами, включая анализ анизотропии

Работа выполнена при финансовой поддержке РФФИ (гранты № 16-02-00488_а и № 16-08-00618_а).

*Московский государственный технический университет имени Н. Э. Баумана, Москва, Россия. E-mail: ingvor@inbox.ru 
микроволнового фона, кластеризации галактик и обилия гравитационно связанных объектов [7]. Также возмущения скалярного поля на инфляционной стадии приводят к появлению гравитационных волн [7].

Возможность того, что каноническое скалярное поле (квинтэссенция) может определять наблюдаемое космическое ускорение, была исследована в работе [8]. В отличие от моделей Вселенной с космологической постоянной $\Lambda$, поле квинтэссенции изменяется с течением времени [9]. Чтобы объяснить наблюдаемое ускоренное расширение Вселенной, в рамках теории гравитации, определяемой действием Эйнштейна-Гильберта, также были предложены модели, которые включают в себя $k$-эссенцию [10], модели, в которых темная энергия взаимодействует с темной материей [11], объединенные модели темной материи и темной энергии [12], космологические модели с фантомными полями [13] и т. д.

Рассмотрим уравнения динамики скалярного поля в случае Вселенной ФридманаРобертсона-Уокера (ФРУ) в системе единиц $c=M_{\mathrm{P}}=1$ на стадии космологической инфляции с каноническим лагранжианом $\mathcal{L}=\dot{\phi}^{2} / 2-V(\phi)$. Для плоской Вселенной ФРУ уравнения записываются в виде [6]

$$
\begin{gathered}
3 H^{2}=\frac{1}{2} \dot{\phi}^{2}+V(\phi), \\
\ddot{\phi}+3 H \dot{\phi}+\frac{d V(\phi)}{d \phi}=0 .
\end{gathered}
$$

Следствием этой системы является уравнение

$$
\dot{H}=-\frac{1}{2} \dot{\phi}^{2}
$$

Для построения непротиворечивой модели космологической инфляции необходимо выполнение следующих условий:

- модель описывает стадию ускоренного расширения, которая подразумевает, что $-1<w<-1 / 3$

- стадия ускоренного расширения завершается вторичным разогревом с последующим образованием фотонов, т. е. переходом на стадию преобладания излучения, которой соответствует равенство $w=1 / 3$;

- полученные в рамках модели параметры космологических возмущений соответствуют наблюдениям.

Для изучения динамики инфляции обычным методом является приближение медленного скатывания, которое подразумевает упрощение уравнений динамики скалярного поля посредством игнорирования вклада его кинетической энергии. В приближении медленного скатывания $\dot{\phi}^{2} / 2 \approx 0$, что ограничивает разнообразие форм потенциала и обеспечивает наличие стадии космологической инфляции.

Исходя из уравнения (3), получаем, что $\dot{H} \approx 0$, т. е. следствием приближения медленного скатывания является условие $H \approx$ const, определяющее ускоренное расширение Вселенной. Записывая параметр состояния в случае эволюции скалярного поля, приходим к равенству

$$
w=\frac{\dot{\phi}^{2} / 2-V}{\dot{\phi}^{2} / 2+V} \approx-1 .
$$


Если не использовать приближение медленного скатывания, то для учета вклада кинетической энергии скалярного поля следует, как правило, также наложить условия на форму потенциала.

В работе [14] рассматривался случай совершенно плоского потенциала $V^{\prime}=0$, который тоже обеспечивает существование стадии ускоренного расширения. В работах [15], [16] проведено обобщение этого случая на случай динамики скалярного поля, описываемой уравнениями

$$
\begin{gathered}
3 H^{2}=\frac{1}{2} \dot{\phi}^{2}+V(\phi), \\
\dot{H}=-\frac{1}{2} \dot{\phi}^{2}, \\
\ddot{\phi}=-(3+\alpha) H \dot{\phi},
\end{gathered}
$$

где постоянная $\alpha$ определяет отклонение от модели с $V^{\prime}=0$, а штрих означает производную по полю.

В моделях $k$-эссенции рассматривается лагранжиан $\mathcal{L}(X, \phi)=K(X)-V(\phi)$, где $X=\dot{\phi}^{2} / 2$, а $K(X)$ - некоторая функция кинетической энергии скалярного поля. В таких моделях решения можно найти для двух стадий: $V(\phi) \gg K(X)$ и соответственно $V(\phi) \ll K(X)$, т. е. для стадии преобладания потенциальной и кинетической энергии [17] или соответственно стадии, когда инфляция обусловлена только кинетической энергией скалярного поля $V(\phi)=0$ [18].

Точные решения уравнений эволюции скалярного поля можно получить с помощью различных методов [19]. Одним из них является метод, впервые рассмотренный Ивановым [20], впоследствии - Салопеком и Бондом [21], который заключается в записи этих уравнений в следующей форме:

$$
V(\phi)=3 H^{2}(\phi)-2 H^{\prime}(\phi)^{2} .
$$

Задавая параметр Хаббла как функцию скалярного поля $H=H(\phi)$, из уравнений (3) и (8) получаем точные выражения для потенциала, поля и масштабного фактора.

В случае точных решений, для которых ограничения на форму потенциала отсутствуют [22], требуется обоснование наличия стадии ускоренного расширения и выхода из инфляции исходя из вида масштабного фактора или параметра состояния [23]. Наиболее очевидным методом построения модели, соответствующей условию перехода от инфляционной стадии к стадии преобладания излучения, является непосредственное задание параметра состояния $w=w(t)$ и последующее вычисление потенциала, скалярного поля и масштабного фактора. Из уравнений (1)-(3) получаем равенство

$$
w(t)=-1-\frac{2}{3} \frac{\dot{H}(t)}{H^{2}(t)} .
$$

Тем не менее точное решение уравнения (9) для подходящей функции $w=w(t)$ не всегда можно найти, решение записывается в квадратурах, и нельзя утверждать, что полученный потенциал $V=V(\phi)$ приводит к реализации механизма генерации элементарных частиц при завершении инфляционной стадии. 
В настоящей работе рассматривается возможность решения уравнений динамики скалярного поля по завершении инфляционной стадии для канонического лагранжиана при отсутствии условий на форму потенциалов и кинетическую энергию. Соответствие наблюдаемым ограничениям на параметры космологических возмущений получается посредством выбора параметров модели.

\section{2. КИНЕТИЧЕСКОЕ ПРИБЛИЖЕНИЕ}

Запишем уравнения (1)-(3) в следующем виде:

$$
\begin{gathered}
3 H^{2}=X+V(\phi), \\
3 H \sqrt{2 X}=\frac{d}{d \phi}(X+V(\phi)), \\
\dot{H}=-X,
\end{gathered}
$$

с учетом соотношений $\ddot{\phi}=\frac{1}{2} \frac{d \dot{\phi}^{2}}{d \phi}$ и $\dot{\phi}=-\sqrt{2 X}$.

Также запишем плотность энергии и давление:

$$
\begin{aligned}
& \varepsilon=\frac{1}{2} \dot{\phi}^{2}+V=X+V, \\
& p=\frac{1}{2} \dot{\phi}^{2}-V=X-V .
\end{aligned}
$$

Зададим кинетическую энергию скалярного поля $X$ как линейную функцию параметра состояния $w$ таким образом, чтобы при $w=-1$ выполнялось условие $X=0$, которое соответствует ускоренному расширению, обусловленному космологической постоянной, т. е.

$$
X=\beta(w+1)
$$

где $\beta$ - положительная постоянная.

Запишем параметр состояния как

$$
w=\frac{X-V}{X+V} .
$$

Подставляя $w$ в уравнение (15), с учетом уравнения (10) получаем, что

$$
3 H^{2}=X+V=2 \beta, \quad H=\text { const } .
$$

Следовательно, определение кинетической энергии скалярного поля в виде $X \approx$ $\beta(w+1)$, которое назовем кинетическим приближением, обеспечивает ускоренное расширение $H \approx$ const и дает возможность нахождения решений уравнений динамики скалярных полей посредством соответствующего выбора параметра состояния.

\section{3. ПАРАМЕТР СОСТОЯНИЯ КАК ФУНКЦИЯ ВРЕМЕНИ}

Рассмотрим параметр состояния следующего вида:

$$
w(t)=\frac{A}{B e^{-\alpha\left(t-t_{\mathrm{e}}\right)}+1}-1, \quad A=\frac{4}{3},
$$




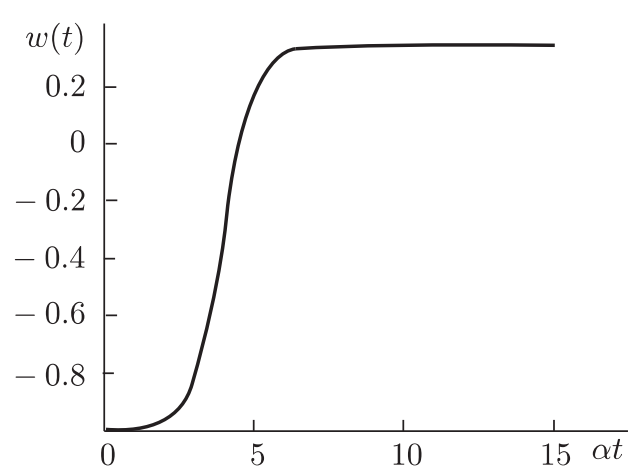

a

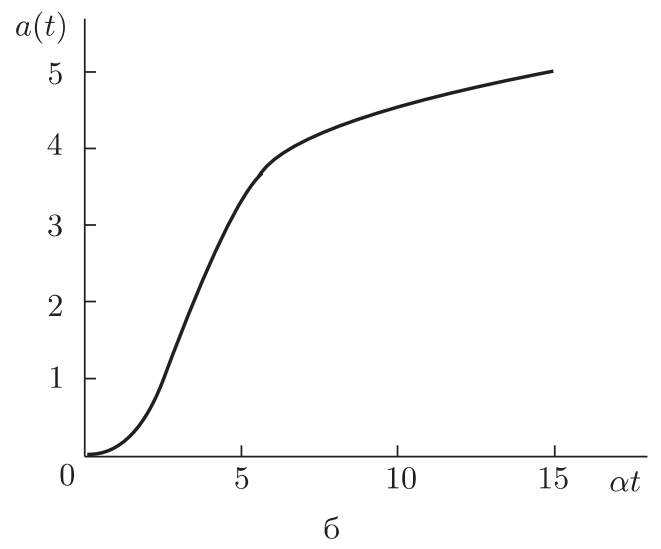

Рис. 1. Зависимости параметра состояния $w=w(t)$ (а) и масштабного фактора $a=a(t)$ (б) от времени для $A=4 / 3, \beta=2.2, H_{0}=2 \times 10^{-5}, \alpha=0.5$ и $B=5 \times 10^{11}$. Масштабный фактор нормирован, $\alpha t_{\mathrm{e}}=4$. После фазового перехода к стадии преобладания излучения экспоненциальное расширение сменяется степенным.

где $t_{\mathrm{e}}$ - время завершения инфляционной стадии, которое определяется условием $w=-1 / 3$. Запишем также кинетическую энергию скалярного поля в виде

$$
X(t)=\frac{A \beta}{B e^{-\alpha\left(t-t_{\mathrm{e}}\right)}+1} .
$$

Из уравнения (15) получаем следующую зависимость скалярного поля от времени:

$$
\varphi(t)=\phi(t)-C= \pm \frac{2 \sqrt{2 A \beta}}{\alpha} \operatorname{arcth} \sqrt{1+B e^{-\alpha\left(t-t_{\mathrm{e}}\right)}},
$$

где $C$ - постоянная интегрирования. Из уравнения (12) находим параметр Хаббла

$$
H(t)=C_{1}+\frac{A \beta}{\alpha} \ln \left(B+e^{\alpha\left(t-t_{\mathrm{e}}\right)}\right),
$$

где $C_{1}=H_{0}-(A \beta \ln B) / \alpha$. Масштабный фактор имеет вид

$$
a(t)=a_{0} \exp \left[C_{1} t-\frac{A \beta}{\alpha^{2}} f\left(1+B e^{\alpha\left(t-t_{\mathrm{e}}\right)}\right)\right],
$$

где функция $f$ определяется следующим образом:

$$
f(\xi)=\int_{1}^{\xi} \frac{\ln \xi}{1-\xi} d \xi
$$

Примеры зависимостей (18), (22) см. на рис. 1.

Запишем параметр Хаббла как функцию скалярного поля:

$$
H(\phi)=H_{0}+\frac{A \beta}{\alpha} \ln \left[\operatorname{ch}^{2} \frac{\alpha \varphi}{2 \sqrt{2 A \beta}}+1\right] .
$$



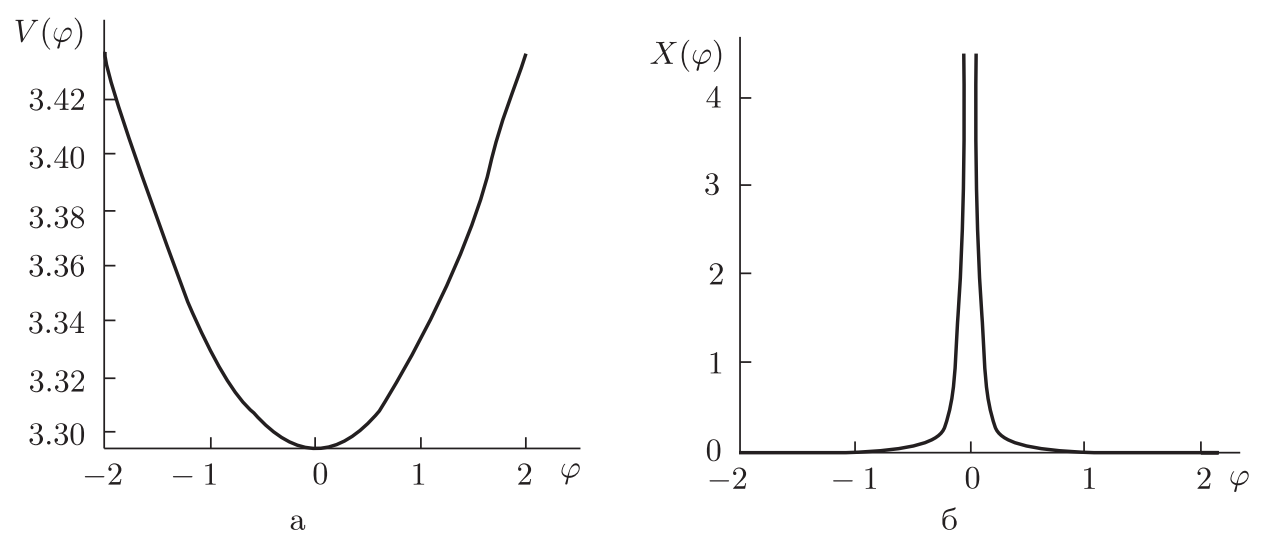

Рис. 2. Зависимости потенциала скалярного поля $V=V(\varphi)$ и его кинетической энергии $X=X(\varphi)$ для заданных параметров. Резкий рост кинетической энергии происходит при осцилляциях поля около минимума потенциала.

Из уравнения (8) получаем потенциал

$$
V(\varphi)=3\left(H_{0}+\frac{A \beta}{\alpha} \ln \left[\operatorname{ch}^{2} \frac{\alpha \varphi}{2 \sqrt{2 A \beta}}+1\right]\right)^{2}+\frac{\frac{A \beta}{4 \alpha^{2}} \operatorname{sh}^{2} \frac{\alpha \varphi}{\sqrt{2 A \beta}}}{\left[\operatorname{ch}^{2} \frac{\alpha \varphi}{2 \sqrt{2 A \beta}}+1\right]^{2}}
$$

Также запишем кинетическую энергию как функцию скалярного поля:

$$
X(\varphi)=\frac{A \beta}{\operatorname{th}^{2} \frac{\alpha \varphi}{2 \sqrt{2 A \beta}}} .
$$

Примеры зависимостей (25), (26) см. на рис. 2.

В настоящее время наряду с другими моделями рассматриваются несколько типов моделей космологической инфляции, которые различаются как видом потенциала, так и начальными условиями, при которых возникает инфляционная стадия: скалярное поле может находиться в одном из минимумов своего потенциала или же ускоренное расширение происходит не в минимуме, а при любых условиях, допускающих возникновение инфляции при значениях плотности энергии скалярного поля, сравнимых с планковскими [6].

Возможны различные режимы развития стадии ускоренного расширения. Также модели могут различаться условием окончания стадии инфляции. Например, в случае медленного скатывания скалярного поля к минимуму потенциала впоследствии происходят осцилляции поля около минимума. В моделях хаотической инфляции завершение стадии ускоренного расширения происходит, когда плотность энергии скалярного поля уменьшается достаточно для того, чтобы “трение" $3 H \dot{\phi}$ также уменьшалось и скалярное поле начало осциллировать около минимума потенциала. В дальнейшем происходит потеря энергии за счет рождения элементарных частиц с повторным (постинфляционным) нагреванием скалярного поля и последующим установлением термодинамического равновесия [24], [25]. 
В большинстве инфляционных моделей повторное нагревание происходит из-за осцилляций инфлатона, которые приводят к большому количеству коротких периодов образования элементарных частиц [24], [25]. Также рассматривается возможность постинфляционного нагревания за счет спонтанного нарушения симметрии в случае гибридной инфляции (с двумя скалярными полями) [26], и предлагаются модели, в которых образование частиц происходит за счет гравитационных эффектов [27].

Для определения поведения потенциала и скалярного поля после завершения инфляционной стадии рассмотрим следующие условия: $\phi_{0}-\phi \ll 1$ или $\varphi \ll 1$. В результате находим, что

$$
V(\varphi)=V_{1} \varphi^{4}+V_{2} \varphi^{2}+V_{0}+\mathcal{O}\left(\varphi^{6}\right)
$$

где

$$
\begin{gathered}
V_{0}=\frac{3\left[H_{0}-A \beta \ln 2\right]^{2}}{\alpha^{2}}, \\
V_{1}=\frac{1}{\alpha^{2}}\left(\frac{3 H_{0}+3 A \beta \ln 2}{768 A \beta}-\frac{1}{768 A \beta}+\frac{3}{256}\right), \\
V_{2}=\frac{3}{8 \alpha^{2}}\left(H_{0}+A \beta \ln 2-\frac{1}{4}\right) .
\end{gathered}
$$

Задавая различные значения постоянных $\beta, \alpha$ и $H_{0}$, получаем следующие типы потенциалов с учетом $V_{0}$ :

- при $V_{1}=0$ - потенциал $V(\varphi)=V_{2} \varphi^{2}+V_{0}+\mathcal{O}\left(\varphi^{6}\right)$, который рассматривался в работе [28];

- при $V_{2}=0$ - потенциал $V(\varphi)=V_{1} \varphi^{4}+V_{0}+\mathcal{O}\left(\varphi^{6}\right)[29]$;

- при $V_{1}>0, V_{2}<0$ - потенциал Хиггса $V(\varphi)=V_{1} \varphi^{4}-V_{2} \varphi^{2}+V_{0}+\mathcal{O}\left(\varphi^{6}\right)$.

Механизмы образования элементарных частиц после завершения инфляционной стадии для этих потенциалов описаны в монографии [6] и в обзоре [30].

Покажем, что поведение скалярного поля (20) при условии $\phi_{0}-\phi \ll 1$ соответствует полученным потенциалам.

Для этого разложим зависимость $(20)$ в ряд по малому параметру $e^{-\alpha\left(t-t_{\mathrm{e}}\right)}$, в результате для знака "+" имеем выражение

$$
\phi-C \approx \frac{\sqrt{2 A \beta}}{2 \pi \alpha}+\frac{\sqrt{2 A \beta}}{2 \pi \alpha} e^{-\alpha\left(t-t_{\mathrm{e}}\right)} .
$$

Полагая $C=-\sqrt{2 A \beta} / \pi \alpha+\phi_{0}$, где $\phi_{0}=\sqrt{2 A \beta} / 2 \pi \alpha$, записываем соотношение

$$
\phi \approx \phi_{0} e^{-\alpha\left(t-t_{\mathrm{e}}\right)},
$$

которое соответствует динамике скалярного поля для потенциалов $V(\phi)=V_{0} \phi^{4}$ или $V(\phi)=V_{1} \phi^{4}-V_{2} \phi^{2}+V_{0}$ с различными значениями $\phi_{0}$ и $\alpha$. В первом случае вторичный разогрев происходит за счет осцилляций скалярного поля около минимума потенциала [6], [30] и реализуется сценарий хаотической инфляции. Второй случай подразумевает фазовый переход из одного локального минимума в другой с повышением плотности энергии и спонтанным нарушением симметрии [6], [30], или реализуется сценарий новой инфляции [31]. 
Раскладывая зависимость (20) в ряд по малому параметру $t-t_{\mathrm{e}}$ и выбирая знак "+", получаем формулы

$$
\begin{gathered}
\phi \approx C+\frac{4 \sqrt{A \beta}}{\alpha}-\sqrt{A \beta}\left(t-t_{\mathrm{e}}\right), \\
\phi \approx \phi_{0}-\sqrt{A \beta}\left(t-t_{\mathrm{e}}\right), \quad \phi_{0}=C+\frac{4 \sqrt{A \beta}}{\alpha} .
\end{gathered}
$$

Скалярное поле $\phi$ соответствует потенциалу $V(\phi)=V_{0} \phi^{2}$. Для потенциала $V(\phi)=$ $V_{0} \phi^{2}$ повторное нагревание происходит при осцилляциях скалярного поля около минимума в модели хаотической инфляции [6], [30].

Аналогично можно получить зависимости (32) и (34) скалярного поля от времени для потенциалов при условии $\phi_{0}-\phi \ll 1$ непосредственно из уравнений (1)-(3).

Таким образом, резкий рост кинетической энергии на завершении инфляционной стадии для (25) происходит или в сценарии хаотической инфляции (этот механизм реализуется для выбранных параметров модели), или в случае спонтанного нарушения симметрии. Оба случая подразумевают повторное нагревание и последующую генерацию элементарных частиц [6], [24], [25], [31].

\section{4. ПАРАМЕТРЫ КОСМОЛОГИЧЕСКИХ ВОЗМУЩЕНИЙ}

В процессе инфляции квантовые флуктуации скалярного поля создают возмущения метрики. В линейном приближении для фурье-мод скалярных $v_{k}$ и тензорных $u_{k}$ возмущений можно записать уравнения Муханова-Сасаки [32]

$$
\begin{aligned}
& \frac{d^{2} v_{k}}{d \eta^{2}}+\left(k^{2}-\frac{1}{z} \frac{d^{2} z}{d \eta^{2}}\right) v_{k}=0 \\
& \frac{d^{2} u_{k}}{d \eta^{2}}+\left(k^{2}-\frac{1}{a} \frac{d^{2} a}{d \eta^{2}}\right) u_{k}=0
\end{aligned}
$$

где $z=a \dot{\phi} / H, k$ - волновое число, $\eta$ - конформное время.

На основе уравнений (35), (36) можно определить спектры мощности и спектральные индексы скалярных и тензорных возмущений.

В работах [33]-[35] получены формулы для расчета основных космологических параметров на пересечении радиуса Хаббла $(k=a H)$ в случае точных решений уравнений динамики скалярного поля.

Определим параметры космологических возмущений для рассматриваемой модели, полагая, что время пересечения радиуса Хаббла $t_{\mathrm{H}} \approx t_{\mathrm{e}}$; в таком случае $e^{-\alpha\left(t_{\mathrm{H}}-t_{\mathrm{e}}\right)} \approx 1$. Также полагаем, что $B+1 \approx B, \ln (1+1 / B) \approx 0$.

Квадраты амплитуд скалярных и тензорных возмущений имеют вид

$$
\begin{gathered}
\mathcal{P}_{\mathrm{S}}=-\frac{H^{4}}{8 \pi^{2} \dot{H}}=\frac{3 H_{0}^{4} B}{32 \pi^{2} \beta}=2.15 \times 10^{-9} \\
\mathcal{P}_{\mathrm{T}}=\frac{H^{2}}{2 \pi^{2}}=\frac{H_{0}^{2}}{2 \pi^{2}}=1.01 \times 10^{-11}
\end{gathered}
$$


Спектральные индексы скалярных и тензорных возмущений записываются как

$$
\begin{gathered}
n_{\mathrm{S}}-1=\frac{4 \dot{H}-\frac{H \ddot{H}}{\dot{H}}}{\dot{H}+H^{2}}=\frac{-12+\frac{H_{0}^{2} B}{3 \beta}\left(1-\frac{\alpha}{B}\right)}{1-\frac{3 H_{0}^{2} B}{2 \beta}}=-0.029, \\
n_{\mathrm{T}}=\frac{2 \dot{H}}{\dot{H}+H^{2}}=-\frac{\beta}{-4 \beta+3 H_{0}^{2} B}=-0.0058 .
\end{gathered}
$$

Тензорно-скалярное отношение дается выражением

$$
r=-4 \frac{\dot{H}}{H^{2}}=\frac{16 \beta}{3 H_{0}^{2} B}=0.047 .
$$

Запишем соответствующие величинам (37)-(41) измеренные параметры космологических возмущений по данным наблюдений спутника PLANCK [36]:

$$
10^{9} \mathcal{P}_{\mathrm{S}}=2.142 \pm 0.049, \quad \mathcal{P}_{\mathrm{T}}=r \mathcal{P}_{\mathrm{S}}, \quad n_{\mathrm{S}}=0.9667 \pm 0.0040, \quad r<0.113
$$

Также запишем число $e$-фолдов, полагая $t_{0} \approx 0$ :

$$
N=\int_{t_{0}}^{t_{\mathrm{e}}} H(t) d t=\left(\frac{H_{0}}{\alpha}-\frac{A \beta}{\alpha^{3}} \ln B\right) \alpha t_{\mathrm{e}}+\frac{A \beta}{\alpha^{2}}\left[f(1+B)-f\left(1+B e^{\alpha t_{\mathrm{e}}}\right)\right] \approx 61 .
$$

Таким образом, рассматриваемая модель удовлетворяет основанным на наблюдениях ограничениям на значения параметров космологических возмущений и ограничению на число $e$-фолдов $N \geqslant 60$ [30].

\section{5. ПАРАМЕТР СОСТОЯНИЯ КАК ФУНКЦИЯ ПАРАМЕТРА ХАББЛА}

Из предыдущих разделов видно, что если задавать параметр состояния как функцию времени $w=w(t)$, получается потенциал сложного вида. Настолько же громоздкие потенциалы можно получить, используя выражения $w(t)=-1+A \alpha^{2} t^{2} /\left(\alpha^{2} t^{2}+1\right)$ или $w(t)=-1+A \operatorname{th}^{2} \alpha t$, которые определяют естественный выход из инфляции с последующей стадией преобладания излучения.

Вместо этого рассмотрим в контексте кинетического приближения модель космологической инфляции, которую зададим, определяя параметр состояния как функцию параметра Хаббла в уравнении (10)

$$
V=3 H^{2}-\beta[w(H)+1] .
$$

Для параметра состояния $w(H)=-1-2 A^{2} H^{2} / \beta+2 A^{2} \lambda^{2}$ получаем решения уравнений (10)-(12) в виде

$$
\begin{gathered}
\phi(t)=A \ln [\operatorname{th} \lambda t], \\
H(t)=A^{2} \lambda \operatorname{ch} 2 \lambda t, \\
a(t)=a_{0}[\operatorname{sh} 2 \lambda t]^{A^{2} / 2}, \\
V(\phi)=A^{2} \lambda^{2}\left[\left(3 A^{2}-2\right) \operatorname{ch}^{2} \frac{\phi}{A}+2\right],
\end{gathered}
$$

которые рассматривались в работе [37] и были получены другим способом. 
Параметр состояния, отличный от $w(t)=-1-2 \dot{H} / 3 H^{2}$ (см. работу [37]), запишем следующим образом:

$$
w(t)=-1-\frac{2 A^{6} \lambda^{2}}{\beta} \operatorname{ch}^{2} 2 \lambda t+2 A^{2} \lambda^{2} .
$$

Естественный выход из инфляции, вторичный разогрев и дальнейший корректный переход к стадии преобладания излучения $(w=1 / 3)$ происходит при выборе параметра модели $\lambda=\sqrt{2 \beta / 3 A\left(\beta-A^{4}\right)}$, где $\beta>A^{4}$.

Также решения уравнений (10)-(12) для произвольного вида потенциалов можно получить с помощью методов, основанных на задании параметра Хаббла или суперпотенциала $W=X+V$, которые рассматривались в работах [20], [21], [22], [23]. При этом наличие стадии ускоренного расширения обеспечивается посредством кинетического приближения.

\section{6. ЗАКЛЮЧЕНИЕ}

В настоящей работе рассмотрен метод решения уравнений динамики канонических скалярных полей на инфляционной стадии эволюции Вселенной, основанный на представлении кинетической энергии поля в виде линейной функции параметра состояния. Такое представление кинетической энергии вводится для обеспечения наличия стадии ускоренного расширения. В рамках предложенного подхода была построена модель космологической инфляции для заданного параметра состояния с выходом на стадию преобладания излучения.

Также в работе был рассмотрен метод построения моделей космологической инфляции в случае кинетического приближения посредством определения параметра состояния как функции параметра Хаббла $w=w(H)$.

Преимуществом кинетического приближения в сравнении с приближением медленного скатывания является учет кинетической энергии поля и существование стадии инфляции для произвольных потенциалов.

Метод кинетического приближения можно применять и для фантомных полей, для которых кинетическая энергия отрицательна [13]:

$$
\begin{aligned}
& \varepsilon=-\frac{1}{2} \dot{\phi}^{2}+V=-X+V, \\
& p=-\frac{1}{2} \dot{\phi}^{2}-V=-X-V .
\end{aligned}
$$

Параметр состояния определяется следующим образом:

$$
w=\frac{X+V}{X-V} .
$$

Для $X \approx-\beta(w+1)$ параметр Хаббла $H \approx$ const, что соответствует стадии ускоренного расширения.

Динамика фантомных полей в контексте кинетического приближения требует дополнительного анализа и будет рассмотрена в отдельной публикации.

Следует отметить, что рассматриваемый подход подразумевает исследование широкого класса моделей космологической инфляции на основе методов точных решений уравнений эволюции скалярного поля. 
Благодарности. Автор благодарит С. В. Червона за обсуждения и комментарии по поводу данной работы.

\section{Список литературы}

[1] A. A. Starobinsky, "A new type of isotropic cosmological models without singularity", Phys. Lett. B., 91:1 (1980), 99-102.

[2] A. H. Guth, "Inflationary universe: a possible solution to the horizon and flatness problems", Phys. Rev. D, 23:2 (1981), 347-356.

[3] S. J. Perlmutter, G. Aldering, G. Goldhaber et al. (The Supernova Cosmology Project), "Measurements of $\Omega$ and $\Lambda$ from 42 high-redshift Supernovae", Astrophys. J., 517 (1999), 565-586, arXiv: astro-ph/9812133; A. G. Riess, A. V. Filippenko, P. Challis et al., "Observational evidence from Supernovae for an accelerating universe and a cosmological constant", Astron. J., 116:3 (1998), 1009, arXiv: astro-ph/9805201.

[4] A. G. Riess, L. G. Strolger, J. Tonry et al., "Type Ia supernova discoveries at $z>1$ from the Hubble Space Telescope: evidence for past deceleration and constraints on dark energy evolution", Astrophys. J., 607:2 (2004), 665-687, arXiv: astro-ph/0402512.

[5] D. N. Spergel, L. Verde, H. V. Peiris et al., "First-year Wilkinson Microwave Anisotropy Probe (WMAP) observations: determination of cosmological parameters", Astrophys. J. Suppl., 148:2 (2003), 175-194, arXiv: astro-ph/0302209.

[6] A. D. Linde, "Inflationary cosmology", Particle Physics and Inflationary Cosmology, Proceedings of the First International Symposium (Northeastern University, Boston, MA, March 27-31, 1990), eds. P. Nath, S. Reucroft, World Sci., Singapore, 1990, 635-656.

[7] A. R. Liddle, "Inflation and the cosmic microwave background", Phys. Rep., 307:1-4 (1998), 53-60, arXiv: astro-ph/9801148.

[8] P. J. E. Peebles, A. Vilenkin, "Quintessential inflation", Phys. Rep. D., 59:6 (1999), 063505, 6 pp., arXiv: astro-ph/9810509.

[9] R. R. Caldwell, R. Dave, P. J. Steinhardt, "Cosmological imprint of an energy component with general equation of state", Phys. Rev. Lett., 80 (1998), 1582-1585, arXiv: astro-ph/ 9708069.

[10] C. Armendáriz-Picón, T. Damour, V. Mukhanov, "k-Inflation", Phys. Lett. B., 458:2-3 (1999), 209-218, arXiv: hep-th/9904075.

[11] C. G. Böhmer, G. Caldera-Cabral, R. Lazkoz, R. Maartens, "Dynamics of dark energy with a coupling to dark matter", Phys. Rev. D, 78:2 (2008), 023505, 8 pp., arXiv: 0801.1565.

[12] A. Y. Kamenshchik, U. Moschella, V. Pasquier, "An alternative to quintessence", Phys. Lett. B., 511:2-4 (2001), 265-268, arXiv: gr-qc/0103004.

[13] P. Singh, M. Sami, N. Dadhich, "Cosmological dynamics of a phantom field", Phys. Rev. D, 68:2 (2003), 023522, 7 pp., arXiv: hep-th/0305110.

[14] N.C. Tsamis, R.P. Woodard, "Improved estimates of cosmological perturbations", Phys. Rev. D, 69:8 (2004), 084005, 14 pp., arXiv: astro-ph/0307463.

[15] J. Martin, H. Motohashi, T. Suyama, "Ultra slow-roll inflation and the non-Gaussianity consistency relation", Phys. Rev. D, 87:2 (2013), 023514, 10 pp., arXiv: 1211.0083.

[16] H. Motohashi, A. A. Starobinsky, J. Yokoyama, "Inflation with a constant rate of roll", JCAP, 09 (2015), 018, arXiv: 1411.5021.

[17] N. Bose, A.S. Majumdar, " $k$-essence model of inflation, dark matter, and dark energy", Phys. Rev. D, 79:10 (2009), 103517, 7 pp., arXiv: 0812.4131.

[18] T. Chiba, T. Okabe, M. Yamaguchi, "Kinetically driven quintessence", Phys. Rev. D, 62:2 (2000), 023511, 8 pp., arXiv: astro-ph/9912463.

[19] A. T. Kruger, J. W. Norbury, "Another exact inflationary solution", Phys. Rev. D, 61:8 (2000), 087303, 4 pp., arXiv: gr-qc/0004039.

[20] Г. Г. Иванов, Изв. вузов. Сер. Физика, 12 (1980), 22-25. 
[21] D.S. Salopek, J.R. Bond, "Nonlinear evolution of long-wavelength metric fluctuations in inflationary models", Phys. Rev. D, 42:12 (1990), 3936-3962.

[22] S. V. Chervon, "Inflationary cosmological models without restrictions on a scalar field potential", Gen. Relativ. Gravit., 36:7 (2004), 1547-1553.

[23] А. В. Юров, В. А. Юров, С. В. Червон, М. Сами, "Потенциал полной энергии как суперпотенциал в интегрируемых космологических моделях", ТМФ, 166:2 (2011), 299-311.

[24] L. Kofman, A. Linde, A. A. Starobinsky, "Reheating after Inflation", Phys. Rev. Lett., 73:24 (1994), 3195-3198, arXiv: hep-th/9405187.

[25] J. García-Bellido, "20+ years of inflation”, Nuc. Phys. B Proc. Suppl., 114 (2003), 13-26.

[26] G. Felder, J. García-Bellido, P. B. Greene, L. Kofman, A. Linde, I. Tkachev, "Dynamics of symmetry breaking and tachyonic preheating", Phys. Rev. Lett., 87:1 (2001), 011601, 4 pp., arXiv: hep-ph/0012142.

[27] G. Felder, L. Kofman, A. Linde, "Inflation and preheating in nonoscillatory models", Phys. Rev.D, 60:10 (1999), 103505, 10 pp., arXiv: hep-ph/9903350.

[28] L. A. Ureña-López, M. J. Reyes-Ibarra, "On the dynamics of a quadratic scalar field potential", Internat. J. Modern Phys. D, 18:4 (2009), 621-634, arXiv: 0709.3996.

[29] T. J. Zhang, Y. G. Shen, "Quantum cosmology with a complex $\phi^{4}$ field at finite temperature", Internat. J. Theor. Phys., 38:7 (1999), 1969-1979.

[30] A. Linde, "Inflationary cosmology", Inflationary Cosmology, Lecture Notes in Physics, 738, eds. M. Lemoine, J. Martin, P. Peter, Springer, Berlin, 2008, 1-54, arXiv: 0705.0164.

[31] A. Albrecht, P. Steinhardt, "Cosmology for grand unified theories with radiatively induced symmetry breaking", Phys. Rev. Lett., 48:17 (1982), 1220-1223.

[32] V.F. Mukhanov, H. A. Feldman, R. H. Brandenberger, "Theory of cosmological perturbations", Phys. Rep., 215:5-6 (1992), 203-333.

[33] S. V. Chervon, M. Novello, R. Triay, "Exact cosmology and specification of an inflationary scenario", Gravit. Cosmol., 11:4(44) (2005), 329-332.

[34] S. V. Chervon, I. V. Fomin, "On calculation of the cosmological parameters in exact models of inflation", Gravit. Cosmol., 14:2 (2008), 163-167.

[35] С. В. Червон, И. В. Фомин, "Квантовое рождение начальных космологических возмущений”, Изв. вузов (Поволжский регион). Физ.-матем. науки, 2008, № 4, 97-107.

[36] P. A. R. Ade, N. Aghanim, M. Arnaud et al. [Planck Collab.], "Planck 2015 results. XIII. Cosmological parameters", Astron. Astrophys., 594 (2016), A13, 63 pp., arXiv: 1502.01589 .

[37] J.D. Barrow, "Exact inflationary universes with potential minima", Phys. Rev. D, 49:6 (1994), 3055-3058.

Поступила в редакцию 26.02.2016, после доработки 14.03.2016 